\title{
Role of the Korea National Institute of Health in the children's health effect study of particulate matter
}

Young Youl Kim, PhD

Division of Allergy and Chronic Respiratory Disease Research, Department of Chronic Disease Convergence Research, Korea National Institute of Health, Korea Disease Control \& Prevention Agency, Cheongju, Korea

\section{Key message}

Particulate matter (PM) adversely affects children's lungs and cognitive development, and an early exposure to it can lead to chronic metabolic diseases. The Korea National Institute of Health convened a Task Force on the Health Impact of PM to produce scientific evidence and promote technological developments to prevent PM-induced health effects.

Children are especially vulnerable to air pollution due to the developing nature of their organs and immune system and their smaller bodies and airways. ${ }^{1)}$ In proportion to their size, children eat more food, drink more water, and breathe more air than adults. ${ }^{1)}$ Numerous studies have shown that exposure to particulate matter (PM) 2.5 negatively affects pregnancy outcomes and has significant implications for public health given the widespread exposure to air pollution and the association of low birth weight or preterm birth with long-term sequelae, such as developmental disorders and chronic lung disease. ${ }^{2)}$ Neuropsychological delays are associated with exposure to surrounding pollutants during pregnancy or childhood. As a source of oxidative stress and inflammatory factors, PM has been proposed to affect the central nervous system by inducing neurological developmental disorders and behavioral changes. ${ }^{3)}$ Asthma, the most common chronic respiratory disease in children, affects numerous people worldwide. ${ }^{4)}$ Accumulating evidence suggests that exposure to high PM levels, either acutely or chronically, is associated with the exacerbation and incidence of pediatric asthma. ${ }^{5)}$ While there is increasing evidence of the impact of chemical, biological, and social environmental factors on children's development, scientific evidence demonstrating how to minimize their adverse health effects is lacking.

Public interest in air quality, including PM, has exploded over the past 5 years, making it a top concern. The Korean Ministry of Trade, Industry, and Energy and the Ministry of Environment have made research and development $(R \& D)$ efforts to reduce $\mathrm{PM}$, and interest is growing regarding how to minimize its adverse health effects. A few studies have established evidence-based strategies for minimizing the health effects of PM in vulnerable

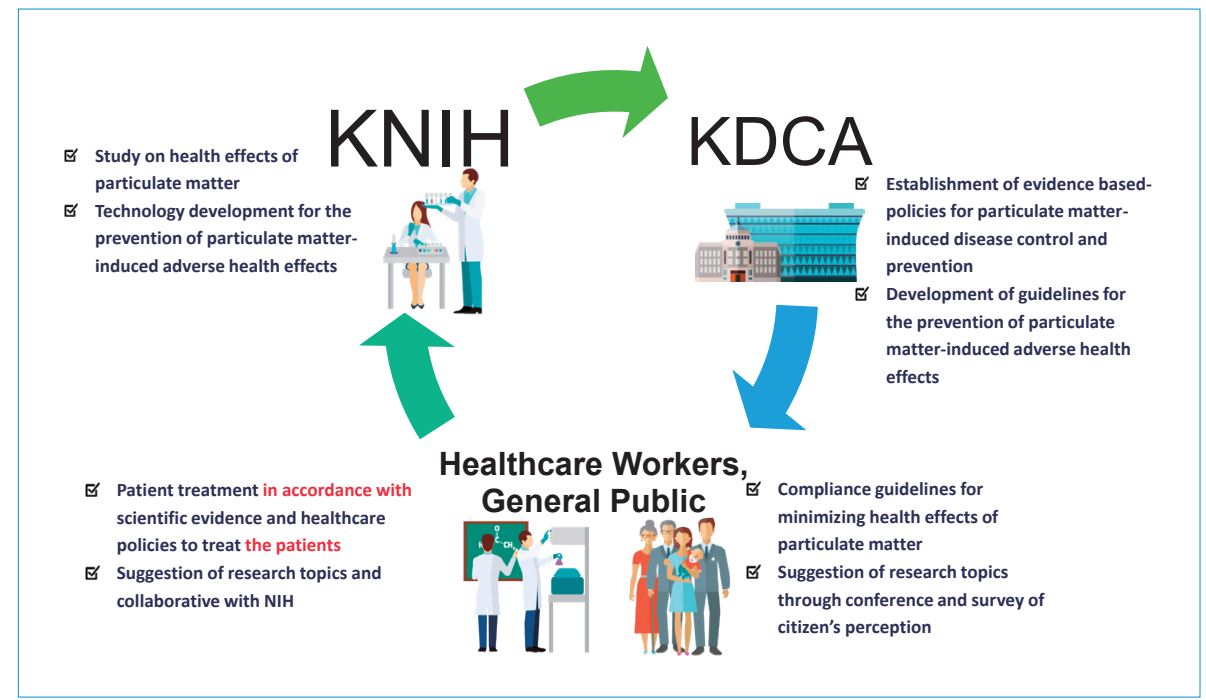

Graphical abstract. The role of the KNIH for the Children's Health Effect Study of particulate matter. $\mathrm{KNIH}$, Korea National Institute of Health; KDCA, Korea Disease Control \& Prevention Agency; KCDC, Korea Centers for Disease Control and Prevention; $\mathrm{NIH}$, National Institutes of Health.

Corresponding author: Young Youl Kim, PhD. Division of Allergy and Chronic Respiratory Disease Research, Department of Chronic Disease Convergence Research, Korea National Institute of Health, Korea Disease Control \& Prevention Agency, 187 Osongsaengmyeong2-ro, Osong-eup Heungdeok-gu, Cheongju 28159, Korea 凶Email: youngyk@nih.go.kr, https://orcid.org/0000-0002-8561-6263

Received: 29 April, 2020, Revised: 2 September, 2020, Accepted: 7 September, 2020

This is an open-access article distributed under the terms of the Creative Commons Attribution Non-Commercial License (http://creativecommons.org/licenses/by$\mathrm{nc} / 4.0 /$ ) which permits unrestricted non-commercial use, distribution, and reproduction in any medium, provided the original work is properly cited.

Copyright (c) 2021 by The Korean Pediatric Society 
populations (children, the elderly, and pregnant women). In response, the Korea National Institute of Health $(\mathrm{KNIH})$ established a governance framework between policy and research and provided a future direction for R\&D for examining the health effects of PM. Through a systematic literature review, the $\mathrm{KNIH}$ established the known health effects of PM, examined research trends for PM, and prioritized research committees on topics including cardiovascular disease, respiratory disease, and vulnerable populations. Each committee selected a major topic and held a forum on research into the health effects of PM. Surveys were conducted to investigate what Korean citizens want to know about PM, while focus group interviews were conducted to investigate what vulnerable populations want to know about PM. This research program aimed to produce scientific evidence for establishing policies and guidelines needed to reduce the adverse health effects of PM.

The main research projects will include: (1) analyzing each population group exposed to PM and building research infrastructure; (2) assessing the adverse health effects of PM in patients and vulnerable populations; and (3) developing technologies for preventing PM-induced adverse health effects. In particular, we will concentrate on evaluating the effects of PM exposure on the exacerbation of childhood asthma using personal monitoring devices and establish an intervention plan for minimizing PMinduced deterioration of childhood asthma based on a patient's estimated personal risk level. According to each child's specific PM exposure level, questionnaires, history, physical examination findings, and pulmonary function and other clinical tests related to respiratory diseases, the effect of PM on lung function and respiratory symptoms in children will be evaluated and preventive guidelines will be provided to decrease their respiratory health problems.

The KNIH will prepare a long-term plan to study the health effects of PM and propose a request for proposals for research that considers the needs of Korean citizens. A governance framework between policy and research will be structured to reduce PM exposure through education and awareness of the hazards of PM exposure and the inherent vulnerabilities of and potential health effects on children.

Conflicts of interest

No potential conflict of interest relevant to this article was reported.

\section{References}

1. World Health Organization (WHO). Children's Environmental Health. Don't pollute my future! The impact of the environment on children's health [Internet]. Geneva (Switzerland): WHO, 2017 [cited 2020 Apr 27]. Available from: https://apps.who.int/iris/bitstream/handle/10665/ 254678/WHO-FWC-IHE-17.01-eng.pdf;jsessionid=3E82373BABB32 D1A6E51274F1DBAA7E7? sequence=1.

2. Li X, Huang S, Jiao A, Yang X, Yun J, Wang Y. Association between ambient fine particulate matter and preterm birth or term low birth weight: an updated systematic review and meta-analysis. Environ Pollut 2017;227:596-605.

3. Calderón-Garcidueñas L, Kulesza JR, Doty RL, D'Angiulli A, TorresJardón R. Megacities air pollution problems: Mexico City Metropolitan Area critical issues on the central nervous system pediatric impact. Environ Res 2015; 137:157-69.

4. Platts-Mills TA. The allergy epidemics: 1870-2010. J Allergy Clin Immunol 2015;136:3-13.

5. Xu X, Zhang J, Yang X, Zhang Y, Chen Z. The role and potential pathogenic mechanism of particulate matter in childhood asthma: a review and perspective. J Immunol Res 2020;17:1-8.

How to cite this article: Kim YY. Role of the Korea National Institute of Health in the children's health effect study of particulate matter. Clin Exp Pediatr 2021;64:139-40. https://doi. org/ 10.3345/cep.2020.00780 